MATEC Web of Conferences 34, 02010 (2015)

DOI: $10.1051 /$ matecconf/ 20153402010

(c) Owned by the authors, published by EDP Sciences, 2015

\title{
RESEARCH ON DETECTION OF MACHINE STIFFNESS
}

\author{
Li-Jie WANG ${ }^{\text {a }}$, Wei-Chao SHI , De-Kai XU \\ Xi'an University of technology, Xi'an, China
}

\begin{abstract}
Machine tool stiffness is a principal factor affecting machine tool precision, traditional methods can only be used to detect limited categories of machine tools. The paper introduces a new scheme to detect machine tool stiffness on the basis of dynamic detection of machine tool stiffness considering its characteristics and stress state during processing. An experiment conducted in turn-milling machining center CH7516GS indicated by comparison that statics analysis of finite elements matched the experimental result well, which provided precise original data for design of improved machine tool precision and access to design of precision detection equipment for other types of machine tools.
\end{abstract}

\section{Introduction}

Machine tool stiffness is a principal factor affecting machine tool precision, which plays an indispensable role in manufacturing for a state. The stiffness of a machine tool is determined when it is designed and produced and varies as it is used and worn. Improvement in stiffness detection of machine tools can provide users precise data during qualification test and application.

The paper introduces a scheme to detect machine tool stiffness on the basis of dynamic detection of machine tool stiffness considering its characteristics and stress state during processing. With the scheme, the loading force of machine tool stiffness can be detected by more real simulation of cutting force loading to work pieces being processed. Besides, it can realize both static and dynamic loading on a single device and simpler data reading during stiffness detection. As a contrast to existing stiffness detection equipment, an experiment was conducted in turn-milling machining center CH7516GS, of which the result was analyzed through finite elements. It is indicated that the designed scheme can provide complete results of machine tool stiffness distribution by

\footnotetext{
a Li-Jie WANG: wlj_sdrc@163.com
}

an experiment and detailed original data for stiffness design of machine tools.

\section{Machine tool rigidness detection scheme design}

The stiffness detection equipment is designed with functions that don't exist in traditional stiffness loading equipment on the basis of existing segmental-support stiffness loading equipment. Comparing with traditional ones, the designed equipment has the following characteristics: 1) imposing a torque on $\mathrm{Z}$ shaft at the point of strength as actual cutting force imposes a torque on $Z$ shaft; 2) its point of strength shall be capable of changing in $\mathrm{XZ}$ plane and realizing continuous loading force as the point of strength of cutting force can be anywhere on XZ plane during actual cutting while the cutting force is continuous; 3) using piezoelectric elements and oscilloscope for reading and fast, precise and detailed recording of loading force; 4) in the deformation detection scheme, the earth of used as a base point to fix the machine tool deformation sensor; thus machine tools are free from errors from deformation detection of tailstock of spindle. 


\section{Design of hardware in machine tool rigidness detection equipment}

\subsection{Design of loading equipment}

For the purpose of research, the cutting force is defined as the center line between lathe spindle and tailstock sleeve during design of rigidness loading equipment, while the points of strength acts on the middle point a shaft with enough rigidness. When a machine tool is measured under a load with component forces Fc: Fp: Ff being 1:0.4:0.25, it has maximum rigidness. Therefore, the loading equipment doesn't contain a component capable of adjusting the ratio of component forces. Among the three component forces, $\mathrm{Z}$ component force is at a direction opposite to coordinate direction while $X$ and $\mathrm{Y}$ component forces are at a direction same with coordinate direction. Thus the azimuth angle of load is determined as $(-0.4,-1,-0.25)$. The machine tool in the experiment was from turn-milling machining center CH7516GS in Baoji machine Tools, Ltd and can withstand a maximum cutting force of $800 \mathrm{Kg}$. During the loading experiment, the selected load was $500 \mathrm{Kg}$.

The force of the loading equipment is detected by a force sensor in tandem connection to a force transmission device. The sensor, which has a built-in piezoelectric device which makes the measurement easier and the reading more precise and faster, is LTH350 typed sensor (Item No. FSH00344) from FUTEK. It has a stainless structure and is capable of resisting excessively heavy load on the basis of metal film strain test technology.

The loading equipment consists of two parts. The first part is the one with force from cutter saddle. Same to three-dimensional stiffness loading equipment, the sliding table of the machine tool drives the cutter saddle. When the device imposing a force moves, the force is loaded. The other part is the test specimen installed between a three jaw chuck and a tailstock. The test specimen carries the load from the part imposing a force and transmits the load to the spindle and the tailstock. Meanwhile, an acting force is imposed on the sliding table of the body of the machine tool. Fig. 1 shows the lathe loading device model produced with Solid works.

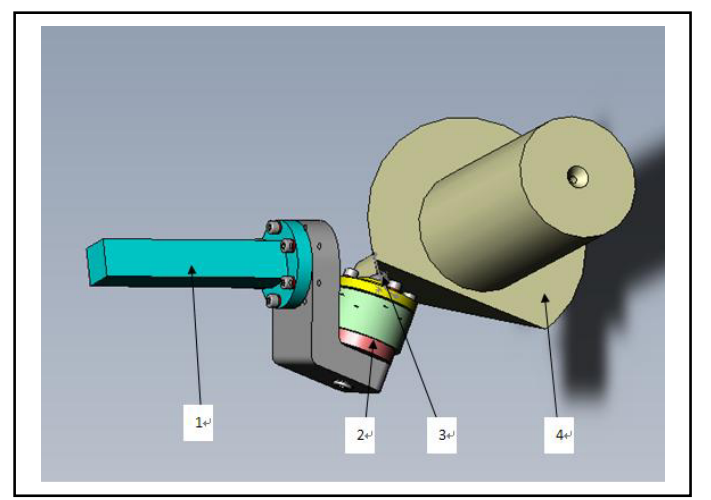

Figure 1. Lathe loading device model

1.the knife holding section 2.the load force sensor 3. Ball 4.specimen

\subsection{Design of lathe micro-displacement detection} equipment.

An electrical-inductance displacement sensor and an eddy sensor were used as micro-displacement detection equipment. The former was used to detect parts with great deformation while the latter is used to detect parts with less deformation. Generally, the displacement of a machine tool is measured by micron and the deformation thickness at less sensitive positions with less deformation is less than 1 micron. In this case, detection equipment must be precisely designed to acquire precise data on deformation of a machine tool.

In the sensor holding device, the ground-based sensor is integrated with the isolation desk where the machine tool is placed. The sensor holding device is comprised of two parts, of which the first one is the holder connected to the vibration isolation desk. The holder has a structure comprised of six main steel frames, on which horizontal and inclined steel pipes with a diameter of $48 \mathrm{~mm}$ and a thickness of $5 \mathrm{~mm}$ are connected. Its designed structure is shown is Fig.2. The other part is the holding part to fix the sensor, where all joints are designed such that six degrees of freedom are adjustable. The sensor holding device is shown in Fig.3.

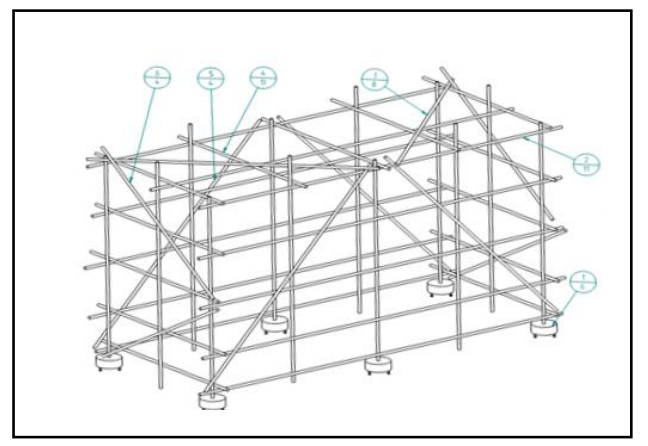

Figure 2. Steel frame structure model

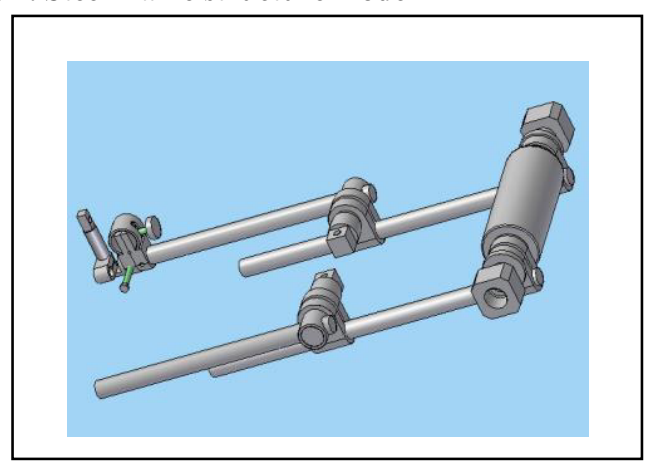

Figure 3. Displacement Sensor holding device

As the sensor holding device used a steel framed structure and a cantilever construction, there are many external interference factors, especially external vibration disturbance and its own vibration due to loading when it is used. These factors shall affect the result of the measure or even bring damages to the sensor due to its contact with the subject. Therefore, the equipment is required to be tested and adjusted after the design is completed. 


\section{Machine tool rigidness detection experiment and data processing}

As one of the core parts of machine tool, the spindle is critical to machining precision, surface quality and production efficiency of the machine tool. As the experiment of rigidness distribution of spindle just emphasizes assessment of the spindle of a machine tool, the deformation of the machine tool itself was not considered during finite element analysis. When the displacement sensor was fixed on basis of the machine tool, the loading position runs through three points: $(108.3265,0.31,184.9),(94.4315,0.31,204.9)$ and (80.527, 0.31, 224.9). Deformation tendency of all loading loads is shown in Fig.4, Fig.5 and Fig.6.

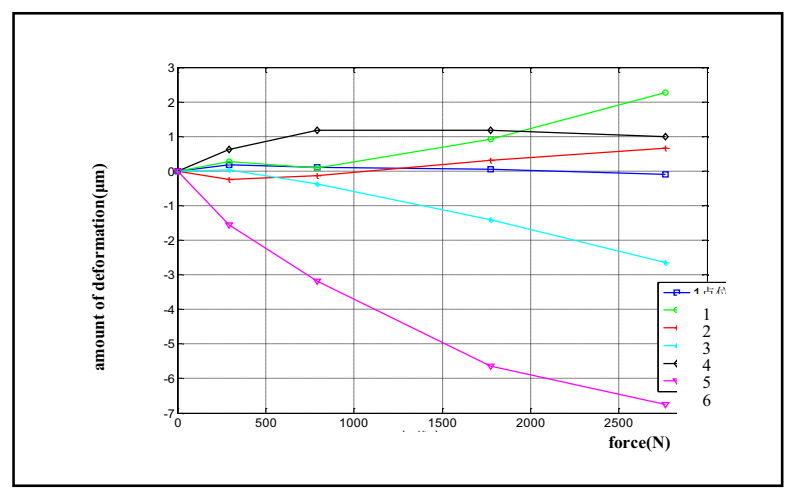

Figure 4. the stiffness curve under first load

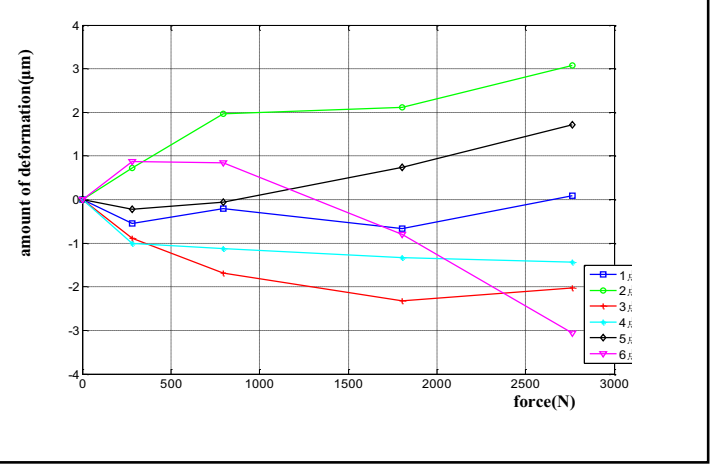

Figure 5. The stiffness curve under second load

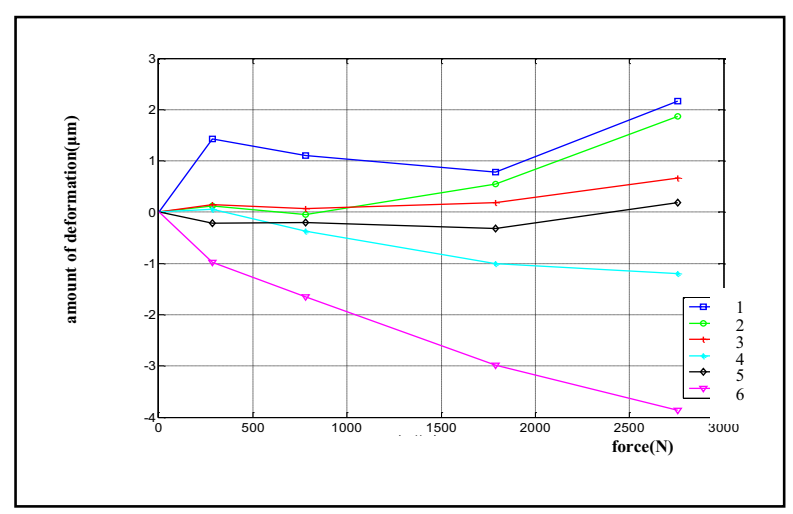

Figure 6. The stiffness curve under third load

The experimental results indicate that the spindle box has less deformation at $\mathrm{X}$ direction with great fluctuation due to unevenness of detection points where the sensor was placed. As the tool is relatively far from the spindle box, a magnetic watch is used as a holding sensor with less rigidness. The data from the detection point 4 of the spindle box at $\mathrm{Y}$ direction indicate that the point was deforming at positive $\mathrm{Y}$ direction and was moving towards bottom right with the loading point with increasingly less deformation. The data from the detection point 5 of the spindle box at $\mathrm{Y}$ direction indicate that the point was deforming at negative $Y$ direction and was moving towards bottom right with the loading point with increasingly less deformation. The spindle box indicates obvious deformation at its $Z$ direction with point 6 deforming at positive $Z$ direction and moving towards bottom right with the loading point with increasingly less deformation. Sharing the same deformation tendency with point 6 , point 2 indicates stable deformation at positive $\mathrm{Z}$ direction. The results indicate strong regularity, realizing the expected experimental objective.

\section{ANSYS-based analysis of machine tool rigidness}

During analysis of machine tool rigidness, SOLIDWORKS was used to produce a model, which was introduced to ANSYS Workbench Environment (AWE), software of finite element analysis for static analysis. After definition of material characteristics and selection of element types, a finite element analytical model was generated through mesh generation. Click Solve in Solution module. Model mesh generation and cloud analysis are shown in Fig.7 and Fig.8. Deformation of the spindle box at both $\mathrm{X}, \mathrm{Y}$ and $\mathrm{Z}$ directions under three types of load were analyzed and found to match the experimental results, which verified the preciseness of the dynamic loading equipment.

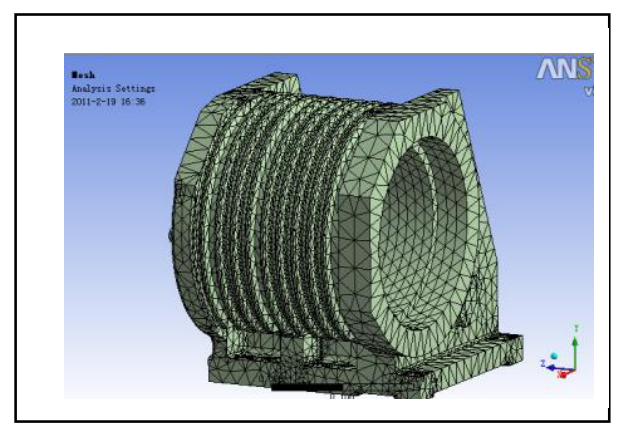

Figure 7. Mesh model

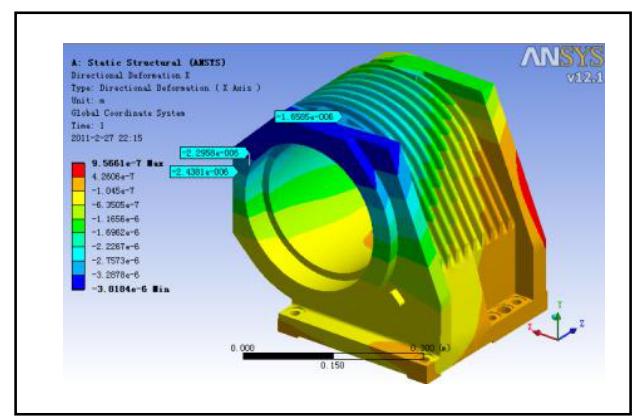


Figure 8.The $\mathrm{X}$ direction of cloud analysis under first load

\section{Conclusions}

The paper introduces a new scheme to detect machine tool stiffness on the basis of dynamic detection of machine tool stiffness considering its characteristics and stress state during processing. An experiment conducted in turning center CH7516GS indicated by comparison that statics analysis of finite elements matched the experimental result well, which provided precise original data for design of improved machine tool precision and access to design of precision detection equipment for other types of machine tools.

\section{References}

1. Huang T, Mei J P. Stiffness Estimation of a Tripodbased Parallel Kinematic Machine [A].Proc of the IEEE Int. Conf Robotic \& Automation [C].2001:3280-3285

2. Bashar S E, Placid M F. Computation of Stiffness and Stiffness Bounds for Parallel Link Manipulators [J].International Journal of Machine Tools and Manufacture ,1999,39(2):321-342

3. P.B.Buchade, A.D.Shaligram. Simulation and Experiment Studies of Inclined Ywo Fiber Displacement Sensor[J].Sensors and Act Uators, 2006,128:312-316

4. H.R.EL-SAYED, Optimum Nose Stiffness of Stepped Machine Tool Spindles [J].Wear 63(1980):231-237.

5. Chow dhury, M.T.Laugier. The Film Stress Measurement by Instrumented Optical Fibrc Displacement Sensor [A].Applied Surface Science, 2007:4289-294

6. Cao Yi, Deng Zhaohui, Xu Huirong. The hardware design and research of $[\mathrm{J}]$. Precision Manufacturing and Automation Test System of Machine Tool Static Stiffness of The Computer, 2010 first, 28-30 